Article

\title{
Technological Socialization and Digital Inclusion: Understanding Digital Literacy Biographies among Young People in Madrid
}

\author{
Daniel Calderón Gómez \\ Department of Social Anthropology and Social Psychology, Complutense University of Madrid, 28223 Madrid, Spain; \\ E-Mail: danielcalderon@ucm.es
}

Submitted: 31 October 2019 | Accepted: 28 February 2020 | Published: 14 May 2020

\begin{abstract}
The main goal of this article is to analyze young people's technological socialization experiences to build a comprehensive model of the distinctive digital literacies interwoven with their biographies. Considering that digital accessibility is a necessary but not sufficient condition for inclusion, we identify which types of digital literacies are linked to the acquisition of digital competencies, confidence, and dispositions towards the incorporation of ICTs into daily activities; on the other hand, we also identify digital literacies that might engender motivated processes of self-exclusion from the digital realm, therefore reinforcing subjects' digital exclusion. Methodologically, this article is based on 30 in-depth biographically-oriented qualitative interviews with young people living in the region of Madrid, Spain. Regarding results, four techno-social dimensions are proposed-motivation, degree of formality, degree of sociality, and type of technological domestication-to construct a typology of four ideal forms of digital literacy: unconscious literacy, self-motivated literacy, professional literacy, and social support. To achieve digital inclusion, self-motivation towards using digital technologies is mandatory, but social practices, academic and professional literacy might work as a secondary socialization process that enhance subjects' affinity with ICTs. Nevertheless, the effect of social support is ambivalent: It could promote digital inclusion among people already interested in digital technologies, but it could also lead to dynamics of self-exclusion among people who are not confident regarding their digital competencies or disinterested in ICTs.
\end{abstract}

\section{Keywords}

digital divide; digital inclusion; digital literacy; technological socialization; young people

\section{Issue}

This article is part of the issue "Digital Inclusion Across the Globe: What Is Being Done to Tackle Digital Inequities?" edited by Bianca C. Reisdorf (University of North Carolina at Charlotte, USA) and Colin Rhinesmith (Simmons University, USA).

(C) 2020 by the author; licensee Cogitatio (Lisbon, Portugal). This article is licensed under a Creative Commons Attribution 4.0 International License (CC BY).

\section{Introduction}

As empirical research on digital inequalities has shown (Lupač, 2018), digital inclusion cannot be achieved just by granting access to digital devices-first-level digital divide-or by increasing the level of digital skillssecond-level digital divide (Hargittai, 2002; van Deursen \& van Dijk, 2014). It is also crucial to generate social spaces of domestication of technology (Silverstone, 1993), focusing on the offline outcomes that people obtain with the use of ICTs - third-level digital divide (Ragnedda, 2017) - and on the incorporated dispositions and experiences of use (Huang, Robinson, \& Cotten, 2015). Regarding this, we will analyze young people's technological socialization experiences to develop a comprehensive model of the influence of digital literacy processes in engendering digital inclusion among young people.

Firstly, digital inclusion can be defined as "the ability of individuals and groups to access and use information and communication technologies" (Institute of Museum and Library Services, University of Washington, \& International City/County Management Association, 2012 , p. 1) which is constrained by the different digital divides that affect people's opportunities to participate in society: Quality of access (Ghobadi \& Ghobadi, 2015), digital skills (Hargittai, 2002), forms of use (van Deursen \& van Dijk, 2013), motivation (van Deursen \& van Dijk, 
2015), emotional barriers (Huang et al., 2015), or offline outcomes and benefits (Ragnedda, 2017) are some of the main dimensions of digital divide research. Thus, digital inclusion and the digital divide are two sides of the same coin, since the digital divide focuses on empirical research about new forms of inequality related to the information society. Reversely, digital inclusion refers to the political intervention among digitally deprived social groups to improve their possibilities to participate in digital society.

Consequently, if participation in digital society is determined by not only material accessibility to digital equipment, services, and tools, but also by subjects' internalized knowledge, digital skills, and dispositions towards technology, to reconstruct people's itineraries of socialization in the use of ICTs, we need to focus on two entangled dimensions: (1) the domestication of technology (Silverstone, 1993), which is related to the particular ways of appropriation and incorporation of digital tools to life, and (2) digital literacies (Erstad, 2011), which refers to the distinctive dispositions, competencies, and attitudes subjectively internalized during the biographical process of domestication of technology. Material and economic aspects can only explain a small part of the many ways in which the Internet is used, which is also associated with the development of particular Internet cultures (Dutton \& Reisdorf, 2019), digital experiences, and attitudes towards the use of digital technologies. It is important to mention, also, the continuities of digital literacies and other forms of cultural literacy (Livingstone, 2008), rejecting the radical split between online and offline spaces of social activity. On the contrary, subjects interact in a digitally mediated world (Lasén \& Casado, 2014) in which the frontiers between online/offline literacies are blurred since cultural background decisively affects people's itineraries of technological domestication and, reversely, the digital skills acquired can be converted into higher levels of cultural capital (Ragnedda, Ruiu, \& Addeo, 2019). Therefore, we need to develop a comprehensive model of digital literacy processes that take into account the differential forms in which people incorporate digital technologies into social spaces of interaction during their lived biographies.

\section{Building a Comprehensive Model of Digital Literacies}

To theorize a comprehensive model of digital literacies, we need to analyze the distinctive dimensions that configure the forms of incorporating digital technologies into daily practice. In this sense, we will analyze subjects' digital literacies taking into account the following dimensions:

A. Temporality: It refers to the duration of the process of acquisition of skills and dispositions since some literacies are concentrated in specific moments of intense use-for instance, conscious learning of specific digital skills in specific social contexts like school or work, whilst others are continuously produced over the course of life through ordinary digital activity. As Robinson (2009) points out, the disinterested use of technology is extremely important in terms of acquiring dispositions and confidence, as it could lead to an informational advantage of those subjects who are more familiar with the use of ICTs.

B. Motivation: In the famous 4-gap model of the digital divide (van Deursen \& van Dijk, 2015), motivation is the first factor that conditions digital appropriation, as skills acquisition is extremely interwoven with motivated forms of incorporating digital technologies into ordinary activity. In such activity, personal dispositions, confidence, and familiarity with the different potentialities of ICTs are also internalized. Hence, it is important to analyze how personal dispositions, interests, and attitudes shape how subjects use digital technologies through their biographical trajectories, engendering particular dynamics of digital literacy that could affect later life stages in terms of digital inclusion. This is important because even if most digital practices are socially mediated, there is always a motivational aspect involved in subjects' digital activity, which is internalized through their biographical socialization in contact with technology.

C. Degree of formality: It refers to the degree of structuration and formalization of the internalized competencies and skills. In this sense, we need to take into account two distinctive forms of literacy (Sefton-Green, Nixon, \& Erstad, 2009): (1) topdown literacy, in which a structured pack of formal skills is proposed by social institutions and organizations as basic competencies needed to get along in the digital era-like e-educational formative programs, and (2) bottom-up literacy, in which the focus is put on micro-social processes of acquiring informal competencies, attitudes, and awareness of the potentialities of digital devices. The first type of literacy is linked to clearly-established digital skills, whilst the second one refers to more informal dispositional competencies and experiences that affect digital practices.

D. Degree of sociality: Despite individual motivation, most digital practices are socially mediated, so personal biographies of socialization on the use of ICTs are also conditioned by the social context of interaction. In this sense, social support (van Deursen, Courtois, \& van Dijk, 2014) is a crucial process through which people can acquire new digital skills and be aware of new features and possibilities of digital devices. Hence, it is important to understand the effect of social resources on engendering digital literacies and, therefore, granting digital inclusion among people, since the specific socio-cultural context in which people are socialized conditions their digital accessibility and digital practices. 
E. Type of devices: Digital literacies are based on specific processes of domestication of technology (Silverstone, 1993), which constitute the objectified material grounds on which personal dispositions can be embodied. In this sense, we will take into account the different technological devices and forms of accessibility present among people and their relation to distinctive forms of literacy. Particularly, the distinction between computeroriented literacy and smartphone-oriented literacy will be considered, since previous research has shown relevant asymmetries between these two forms of accessibility (Pearce \& Rice, 2013).

From the previous five dimensions that condition technological domestication, we have developed an ideal typology of four distinctive forms of digital literacy that can be tracked among subjects' biographical narratives (Table 1). The first two forms (unconscious and selfmotivated literacy) refer mainly to personally-oriented experiences of contact with ICTs, whilst the second two (professional literacy and social support) are much more socially-oriented and depend on social spaces of interaction in which subjects participate. Hereunder we introduce these four literacies and their theoretical articulation, whilst in Section 4 we will develop and discuss them concerning the empirical material.

Type 1. Unconscious literacy: It is the most important form of digital literacy, referring to the involuntary process of incorporating dispositions and competencies during the continuous domestication of technology through life. In terms of temporality, unconscious literacy is a long-term process inherently associated with the use of digital devices at different stages in life, taking into account the different contexts of use in which ICTs are needed. Therefore, the level of motivation is high, but associated with the ordinary use of devices as means for particular ends-what Robinson (2009) calls disinterested forms of use-instead of directly linked to the acquisition of new digital competencies. In contrast, the degree of formality and sociality are generally low, although it is logical to assume that every other form of digital literacy is partially based on this continuous process of internalizing dispositions as structured and structuring structures: in Bourdieu's (1979) view, dispositions derived from so- cial positions of activity but also dispositions as generative principles for new actions. Such dispositions are biographically embodied in long-term processes of social interaction in distinctive social fields in which they are put into practice, so subjects usually deploy them strategically even if they are not aware of it (Kvasny, 2006). As the more generalized form of literacy, unconscious literacy is associated with every technological device: in a long-lasting biographical experience, users learn not only how to technically operate devices but also particular ways in which, in their social worlds, these devices become practically useful.

Type 2. Self-motivated literacy: It is an individualistic literacy in which users consciously try to acquire a specific digital skill or learn how to use digital technologies in a particular way. Hence, self-motivated literacy is generally based on subjects' perceptions of their lack of competency for fulfilling a particular task required in their social life. In these cases, they proactively invest time and effort in learning this concrete digital skill using tutorials, guides, courses or any other formative option. Temporarily motivated literacy is occasional, since it is concentrated in specific moments of intense effort rather than extended over time. The level of motivation required is also high, like in unconscious literacy, but in this case, the focus is placed on the development of new digital skills rather than on the ordinary use of technology. Consequently, the level of formalization of this literacy is extremely high, but the level of sociality could vary: It is high in the case of formative courses and social activities related to digital capacitation, but it is low in the case of personal use of online tutorials and guides. In terms of domestication, this kind of literacy is usually linked with a computer, since the self-perceived lack of digital skills is more common around this device. Computers are usually associated with more productive forms of use (Pearce \& Rice, 2013), so certain users perceive the necessity of fulfilling certain digital tasks that require the use of this equipment but lack the specific knowledge or competencies to do it.

Type 3. Professional literacy: It can be defined as an top-down form of literacy (Sefton-Green et al., 2009) since it refers to social contexts in which the use of digital devices is linked to very formalized prac-

Table 1. Processes of digital literacy.

\begin{tabular}{llllll}
\hline Type of literacy & A. Temporality & B. Motivation & $\begin{array}{l}\text { C. Degree } \\
\text { of formality }\end{array}$ & $\begin{array}{l}\text { D. Degree } \\
\text { of sociality }\end{array}$ & E. Type of devices \\
\hline T.1. Unconscious literacy & Continuous & High & Low & Low & All \\
T2. Self-motivated literacy & Occasional & High & High & Low & Computer \\
T3. Professional literacy & Both & Medium & Medium & High & Computer/Smartphone \\
T4. Socio-interactive literacy & Both & High/Low & Medium & High & Computer/All \\
\hline
\end{tabular}

Source: Own elaboration. 
tices: mainly academic contexts, from school to university, and the labor market. In this case, temporality is ambivalent, since work and the education system are long-term spaces of socialization along with people's biographies but, at the same time, technological domestication and literacy are generally confined to occasional moments of intense use of digital devices. This is why professional literacy requires a medium level of motivation since the level of proactivity and interest in internalizing new digital competencies is generally lower than self-motivated literacy but higher than unconscious literacy, in which the focus is the practice itself rather than the competency. Also, among professional literacy, the level of sociality is high since digital practices are entangled with the requirements of the social contexts in which they are deployed-this is particularly important in the case of the labor market. Finally, like self-motivated literacy, professional literacy is usually linked with the use of computers rather than other devices, although mobile phones are becoming more and more present in some professional ambits.

Type 4. Social support: The last form of literacy, of which the main characteristic is its high degree of sociality, is social support (Courtois \& Verdegem, 2016; van Deursen et al., 2014), which can be defined as one's potential opportunities of taking advantage of acquaintances' digital competencies to deploy digital practices without the knowledge needed to do it autonomously. In sum, it refers to the possibilities of mobilizing social capital to increase the variety of digital tasks that people can fulfill. In terms of temporality, social support is occasional and limited to particular moments in which the necessity of completing a certain task is crucial. In terms of motivation, the situation is ambiguous, since social support can include a high level of motivation in those cases in which subjects are interested in learning new skills or an extremely low level of motivation in the opposite cases, in which subjects are not interested in learning but just in fulfilling a certain task. Regarding the rest of the characteristics of social support, this kind of literacy includes a high level of formality - since the main objective is the specific task itself-and it is linked to all digital devices, although it is extremely relevant in the case of computers.

\section{Methodology}

This article is based on 30 qualitative in-depth interviews carried out in the region of Madrid, Spain, between 2017 and 2018, covering the city of Madrid and localities in the metropolitan area. The sample includes subjects between ages 18 and 35 who use the Internet frequently, so we have not considered in our sample the group of people physically excluded from digital technologies and, therefore, affected by the first-level digital divide (see Mariën \& Prodnik, 2014; Reisdorf \& Groselj, 2017). Therefore, we have developed a structural sample design (Valles, 2014) based on the theoretical representation of three socio-demographic variables: gender, generational position, and educational level. In Table 2 we present the sample design, in which all the crosses between variables have been considered, except for people between ages 18 and 22 with higher education, which is theoretically impossible because of their age.

Gender is an important variable in terms of the second-level digital divide (Antonio \& Tuffley, 2014; Haight, Quan-Haase, \& Corbett, 2014), in which focus lies on the distinctive digital practices developed by users in terms of their motivations, digital skills, and dispositions towards technology. In the Spanish case, this is extremely relevant, since the gender digital divide has been one of the more productive fields of research in recent years (Castaño, Martín, \& Martínez, 2011).

Regarding generational position, as Bolin (2018) points out, dispositions to ICTs acquired during childhood and adolescence can affect later life stages in terms of digital practices, engendering distinctive generational identities that are nevertheless constrained by other socio-economic and cultural conditions. This is why we have divided our sample into three generational groups to compare differential processes of technological socialization among young people during different life stages: ages 18-22, ages 23-29, and ages 30-35.

On educational level, as recent studies demonstrate (Dutton \& Reisdorf, 2019; Haight et al., 2014; Mariën \& Prodnik, 2014), cultural and educational capital are some of the more important variables to explain the secondlevel digital divide, in contrast to economic capital, which is more relevant to understand material accessibility (firstlevel digital divide). This is why we have included in our sample a comparison between 16 subjects with secondary education and 14 subjects with higher education.

Table 2. Qualitative structural sample.

\begin{tabular}{llll}
\hline Educational level & Age group & & \\
& $18-22$ years old & $23-29$ years old & $30-35$ years old \\
\hline Secondary studies & 6 interviews & 9 interviews & 1 interview \\
& $(2$ men; 4 women) & (6 men; 3 women) & ( 1 man; 0 women) \\
Superior studies & 0 interviews & 9 interviews & 5 interviews \\
& $(0$ men; 0 women) & (3 men; 6 women) & (2 men; 3 women) \\
\hline
\end{tabular}

Source: Own elaboration. 
Regarding discourse analysis, we used a sociohermeneutic biographical approach (Alonso, 1998; Wengraf, 2001), which focuses on the pragmatic dimension of language, reconstructing subjects' life stories in the use of digital devices. Hence, we will identify the distinctive ways in which people incorporate ICTs into their practices and the phenomenological internalization of dispositions towards technology.

\section{Results: Digital Literacies as Paths for Digital Inclusion}

Through the qualitative analysis of the interviews, it became evident that digital literacy isn't a homogeneous process since it is closely interwoven with the particular itineraries of technological domestication throughout life. Thus, four distinctive forms of digital literacy have been reconstructed and will be hereunder developed across the subjects' narratives: (1) unconscious literacy, referring to the involuntary acquisition of dispositions during digital practices, (2) self-motivated literacy, referring to the voluntary learning of certain skills, (3) professional literacy, linked to the formally social contexts of use of ICTs, and (4) social support, referring to the mobilization of social capital to improve competencies and fulfill digital tasks.

\subsection{Unconscious Literacy}

I always had the theory that you only learn by doing....By using the Internet, eventually you learn how to use it. I still see people who even nowadays are not familiar with it, but as I have to use things, in the end I learn how to do it. (Man, b. 1988)

Unconscious literacy is the most basic form of digital literacy, related to the internalization of digital competencies, attitudes, and dispositions towards ICTs. This is embedded in technological domestication processes, that is, in quotidian digital practices in which technology is incorporated into personal practices. It has become clear among the interviews that beyond specific digital skills, which are required in certain social contexts, the more important forms of digital literacy are related to the acquisition of familiarity, confidence and pro-technology attitudes, which could engender future process capacitation in the use of such devices and tools. Regarding this, although unconscious literacy is present among all users, whose objectified digital practices are phenomenologically incorporated as dispositions in their habitus, it is also clear that a long-term process of socialization in the use of digital devices for different purposes is crucial to acquiring confidence, familiarity, and awareness of their potentialities. Hence, good quality of access is a necessary but insufficient condition to develop a more flexible and advanced techno-biography in terms of digital literacy. Cultural and social variables are also extremely relevant in acquiring interests and motivation towards the use of new features of the digital world. In the end, as it is shown in the extract below, every user relies on their internalized digital competencies and interests towards digital tools to increase their digital competencies:

I think I use the Internet until I don't know how to do something and, generally, when I can't do something, it's over. I think we are forced to do it yourself logic, you need to fix problems on your own when in the end reality is quite the opposite: no one is an expert on computers. And I also think our resources are scarce, we don't know how to properly use apps or programs such as Excel....I feel it daily, constantly, you have the opportunity to learn new things but you almost always rely on yourself, even if you can look it up on google to learn. (Woman, b. 1993)

In this sense, there is an important divide between those users who have developed a long-term process of socialization in the use of personal computers since childhood and adolescence in comparison with those subjects whose accessibility has mainly been associated with the use of smartphones and other devices. As some authors remark (Lee, Park, \& Hwang, 2015), computer-oriented users usually develop more flexible patterns of use of the Internet than smartphone-oriented users, so having experienced a longer process of computer literacy could become an informational advantage in terms of the offline outcomes and benefits that people obtain from the information society. Thus, the effect of unconscious literacy in digital inclusion is more associated with the familiarization with digital tools and the acquisition of confidence and awareness of their potentialities rather than with learning a certain set of digital skills. The most important competency is the confidence in the possibility of acquiring new digital skills if needed, rather than just the knowledge of a limited set of tasks. This confidence is acquired during long-term processes of familiarization and domestication of technologies-even if they appeared as "wasting" time online, as Robinson (2009) remarked, so digital inclusion policies should take into account the importance of promoting the use of a wider variety of digital devices, even experimenting and investigating new forms of appropriation to particular goals, instead of just teaching a formal set of digital competencies. A good example of this confidence is presented below, in which a respondent admits the importance of his past digital experience during later stages of life:

I started downloading when Edonkey appeared, and after Emule, Napster and so on, more than fifteen years ago. You had to stay for three or four days to download a film, but you learned a lot about the Internet. I was an early adopter, in this sense. This experience of cracking [installing software without a license] and seeking on the Internet helped me a lot afterward. (Man, b. 1984) 
Regarding socio-demographic inequalities among our respondents, the main gender inequalities in terms of unconscious literacy are linked to the longer biographical experience of socialization in the use of digital devices by men, in comparison to women. If this gap is not compensated by alternative forms of literacy (such as professional literacy), the knowledge gap between men and women can become even wider in subjects' future life stages. In terms of generational location, literacy associated with mobile devices (smartphones, tablets, etc.) is predominant, whilst among people between ages 23 and 29 , literacy associated with a personal computers is more common, which could serve as an informational advantage in terms of productive forms of use. Among older respondents, literacy trajectories are much more discontinued, and the gap between pro-technology users and people disinterested in the digital world is much wider. Finally, at the educational level, unconscious literacy is more diverse and extended among subjects who went to university; they developed new forms of domestication of technology after adolescence which could lead to the acquisition of a very diverse technological habitus.

\subsection{Self-Motivated Literacy}

For me, it was never difficult to learn how to use computers; they are quite intuitive. You almost learn by trying, by trial and error, almost crashing the computer, rebooting it and so on. And when I have had a problem or doubt I just needed to look on the Internet, on Google, where you can find everything. (Man, b. 1988)

Besides unconscious literacy, another form of individualized digital literacy is self-motivated literacy, which emerges when people need to fulfill a certain task, but they lack the specific digital competencies to do it, so they voluntarily invest effort and time to acquire the knowledge needed. As remarked in the quote above, selfmotivated literacy is much more common among young people who are familiarized with the use of digital technologies and confident in their potential to acquire new digital skills. Therefore, this kind of literacy is extremely interwoven with pro-technology attitudes and personal interest in the digital world. As a consequence, people who are not confident in their digital competencies or who don't have an interest in digital technologies are in a worse position in terms of taking advantage of the possibilities of the digital realm. They usually don't trust their capacity to learn new skills, which is influenced by their low interest in exploring new features and options of digital devices. Also, in terms of digital inclusion, it is important to remark that motivated literacy, because of its limited temporal focus and high level of formality, is more useful to acquire particular digital competencies needed to fulfill distinctive tasks rather than to internalize general dispositions, familiarity, and confidence in the use of digital technologies. Consequently, formative policies might have a limited effect on digital inclusion if their focus is generally on a specific set of skills-top-down digital literacy-rather than in generating interests and encouraging people to explore and experiment with technology. In the next quote, we reproduce a case of strong motivation towards digital devices, remarked by the necessity of becoming "autonomous" and "self-taught":

In the beginning, there weren't even forums or tutorials, so you had to learn by yourself or ask friends....After forums and other web pages dedicated to specific issues appeared, so information became wider and more independent from offline relationships; you could directly become autonomous and self-taught when you had to learn how to do things. (Man, b. 1984)

Nonetheless, it is important to highlight that selfmotivated literacy could also appear among users who aren't familiar with the use of digital devices. In such cases, respondents aren't interested in digital technologies, but they feel the need to use them, becoming frustrated and hopeless because they think that a huge investment of time and effort is needed to get familiarized with ICTs. Also, it is quite common to turn into their social circle-see Section 4.4-and delegate such digital practices, but when this is impossible, they need to seek online tutorials or formative courses. Thus, formal training courses are much more common among respondents who are unfamiliar or disinterested in digital technologies. Despite this, in our sample, individualized forms of motivated literacy (online guides, tutorials, etc.) are mentioned much more frequently than formal courses or training, which seems to be quite infrequent among young people. A case of this necessity of using digital tools combined with a lack of confidence and interest is reproduced below:

I bought a computer three days ago, and I am bothered about having to learn how to use a new laptop, installing programs, I didn't even have Office yet, because buying it was very expensive and I don't know how to crack it.... have to ask my sister about everything because she is much more skilled than me....If I couldn't rely on her, I would be desperate, since I have no idea about computers. I had my laptop for three days and I still don't know what to do. (Woman, b. 1988)

Taking into account the socio-demographic profile of our sample, an important gender divide emerges, since young men are usually more interested in the use of digital devices-particularly computers-than women. This could engender new digital asymmetries because of the differential technological trajectories of men and women. Therefore, the gender gap is more related to the motivation and emotional costs (Huang et al., 2015) rather than specific digital skills, so digital inclusion poli- 
cies should focus mainly on engendering subjects' confidence and interest in using digital technologies. In terms of generational location, a lack of interest towards the digital realm is more common among older respondents, although younger people are commonly more interested in the use of mobile devices in comparison to personal computers, which are more present among people between ages 23 and 29, a generation closely entangled with the use of computers since adolescence. This is logical, as older generations did not feel the need to use digital devices at all during their primary socialization, whilst younger respondents have been socialized in a muchdiversified landscape of forms of accessibility. Finally, a higher educational level is also generally associated with pro-technology attitudes and higher interest in learning new digital skills; however, this is generally mediated by formal spaces of digital use, which we will develop in the next type (professional literacy).

\subsection{Professional Literacy}

During school, I started to do reports and to use the Internet a little bit more. Afterward, during highschool, we have a specific subject about computing and we learned how to use PowerPoint and Excel. (Woman, b. 1990)

Professional literacy refers to the formally-structured social contexts in which agents interact during their technological socialization: mainly schools, universities, and the labor market, as remarked in the quote above. Some of these spaces are shared by most young people, such as schools, while others depend on their biographical trajectories, such as universities and work. Although school is one of the first spaces of contact with digital technologies after one's house, its incidence in subjects' technodispositions and digital skills is quite low: Respondents remarked that their interest in the digital world usually drifts towards friends and family rather than school, in which digital tools are mainly associated with basic office tasks and Internet information-seeking skills. Nevertheless, the case of universities and work is different, since these two social contexts appear as very important spaces of domestication of technology which allow people to compensate and complement their previous digital skills. In other words, personal use of digital tools during adolescence and childhood can be associated with a primary socialization process, whilst university and work can be conceptualized as secondary socialization instances, allowing those subjects who get to university, or who work in a position related to the use of ICTs, to increase their digital competencies. Below, we include two extracts of interviews in which the entanglement between digital technologies and work position is described:

I work in a hotel, so everything is done by using a computer: sending and receiving emails, using the pro- gram to manage reservations, looking for the information requested by clients, such as tourist guides, tours, an address, or anything. Of course, everything is connected to the Internet, so if there is a problem with the connection we can't work. (Woman, b. 1995)

Understanding the Internet as something connected with many different things, I use it mainly for access to information since my work is linked with data-analysis. I have to look for databases and other information sources, and of course, I have to use email as the principal communicative tool at the office. (Man, b. 1984)

In terms of digital inclusion, professional literacy is extremely important among subjects who haven't developed a long-term process of technological domestication during adolescence. As a secondary form of socialization, professional literacy allows people to develop new dispositions and attitudes towards the use of digital technologies. These new dispositions are not just formal digital skills but attitudes, expectations, and representations of the potential of digital technologies, which become particularly relevant when they are not confined to the specific formal tasks needed at university or in the labor market, but when they are transferred to new spaces of activity. In other words, people who were not interested in the digital world during adolescence-whose unconscious literacy was scarce and fragmented-can internalize new digital dispositions at work (and at university) and transfer them to other daily spaces of activity, increasing their confidence in the use of digital devices. For instance, in the next extract of the interview, we can realize how professional literacy can modify how people represent digital technologies and their relationship to them:

I was hopeless with technology, I have always rejected computers and they were always hard for me. For example, learning how to use Excel was very difficult.... On the one hand, I reject these tools, I am quite narrow-minded regarding them, I am not interested in learning because I am not enthusiastic about technology..... On the other hand, in the end, I was forced to learn because of work; at my job they love Excel and I thought: 'You will have to learn.' I looked for online guides, I asked colleagues for help and, step by step, I more or less managed to get along, since I didn't want to be a burden to them....In the end, you discover it's not so complicated, you just need to invest time and little by little you learn how to deal with such tools. (Woman, b. 1991)

Consequently, there is an important gap between those subjects who went to university and those who did not, since the first ones have experienced a secondary process of domestication added to their previous digital practices, which could place them in a position of informational advantage-the same idea might apply 
to those subjects who have worked on technologicallymediated posts. Also, professional literacy appears as an efficient way of reducing the gender digital divide, since this kind of familiarization with the use of computers at work and university is still more common among women, allowing them to reduce their confidence and emotional gaps (Huang et al., 2015) in comparison to men. Hence, formative policies should include these long-term forms of domestication of technology, oriented toward the familiarization with the use of ICTs after adolescence and, therefore, they should promote the emergence of social spaces in which the use of technology could motivate digitally excluded people.

\subsection{Social Support}

I learned from my brother, since he had much knowledge about computers and liked that world. He was the one who told me: 'When this happens you have to do this.' Many of the tricks I know today are because of him; even if my skills are quite basic, at least I can get along with digital technologies. (Man, b. 1996)

The last form of digital literacy appeared during the interviews is social support, which can be conceptualized as an interpersonal link between two or more subjects in which digital knowledge is shared among the group, so people can take advantage not only of their own digital skills but also of their acquaintances. As highlighted in the quote above, the domestic space is, in many cases, the first space of sharing digital knowledge among family members, even before school, since it is at home where most of the respondents had their first contact with digital technologies. This situation generates an unequal space of possibilities since those subjects who were able to have access to digital devices since childhood and, more importantly, who had a connection to someone who could introduce them in the digital world, experience an informational advantage in terms of familiarization with ICTs. Therefore, the differential social resources are the main form of inequality associated with this form of literacy, but the possibility of taking advantage of such resources depends on other forms of literacy present in people's biographical processes of domestication of technology-particularly unconscious literacy. Hence, engendering social networks of support is a good policy in terms of digital inclusion, but it also requires a personal proactive attitude and motivation to incorporate such potential competencies and avoid just delegating digital practices. In the following extract of an interview, the interconnection between social support and personal motivation is clearly remarked:

In the end, all the apps have options that you don't know how to use. If, for instance, I see someone doing something that I don't know, I ask them: 'Hey, how did you do that?' 'You have to press this arrow and this button.' Then you learn that, but just because you like the platform, if there is something you don't like, you will never learn how to use it. For example, this was my problem with Snapchat, I used to see everybody with dog ears or something but I don't like the app, so I have never had the interest to learn how to do it. (Woman, b. 1994)

Consequently, a crucial issue regarding social support is the ambivalent interconnection between personal motivation towards the use of ICTs and the possibility of delegating certain digital tasks to someone else. As clarified in the quote above, one will only learn how to use a certain tool if he/she is interested in it, so in the cases in which subjects are not motivated to learn how to do something, they will simply ask someone else to do it for them. Such proxy-delegated uses-someone else does the task instead of the subject-are ambivalent in terms of digital empowerment: On the one hand, people can complete tasks which are impossible individually; on the other hand, they do not learn how to complete such tasks, so they become less motivated to incorporate new competencies and their confidence in the use of digital tools is reduced. Consequently, social support cannot be directly transformed into higher digital inclusion, since this inclusion is generally achieved in terms of task resolution, that is, by bypassing the limits of one's internalized competencies through mobilizing social capital to take advantage of others' digital knowledge. In the quote reproduced below, we can find an example of this complete lack of interest in acquiring new digital skills and total digital dependence on the family:

I use a platform recommended by my brother to watch movies online....I don't download anything because my brother does it for me. I am so bad with those things, it's very difficult to find a page to download movies with good quality.... He also helps me if I have a problem with my computer, I know how to use some tools, especially for work, but for anything else, I depend on my brother. (Woman, b. 1982)

Finally, we need to consider the interconnection between social support obtained for using digital technologies and subjects' sociodemographic and cultural conditions. Among our respondents, we found that those subjects who have experienced a long-lasting process of familiarization with the use of digital technologies, particularly computers, since childhood and adolescence are less prone to delegate digital practices to their social ties. On the contrary, they usually act as expert mediators for others, aiding relatives and friends in fulfilling difficult digital tasks. Also, when they need support for a particular task, they are usually motivated to learn how to do it instead of just delegating it, so social support has a positive outcome among them. In terms of gender, in general, more men act as expert mediators for their social circle, although gender differences are nuanced among better-educated people and subjects who 
usually work using computers and digital tools. In generational terms, proxy-delegated uses become much more common among older respondents, whilst younger ones are generally more interested in the digital world and use social support to increase their digital knowledge. Finally, in terms of education, it is clear that universities serve as a secondary form of technological socialization which engenders new interest among digital tools, so bettereducated subjects are generally less dependent on delegated digital practices.

\section{Discussion}

In this article, we have focused on the connection between digital literacies and technological socialization. Broadly, we have developed two individual forms of literacy (unconscious and self-motivated literacy) and two socially-mediated forms of literacy (professional literacy and social support) to conceptualize the particularities of the mechanisms by which people incorporate digital tools into their ordinary practices. Unconscious literacy is present among all users, but those subjects who have experienced longer itineraries of familiarization with the use of ICTs since childhood are generally in a better position, in terms of digital inclusion, to take advantage of the potentialities of the digital world. As a consequence, selfmotivated literacy is especially relevant among those subjects who are already familiar with the use of digital skills, investing time and effort to improve their skills and therefore increasing the gap among digitally excluded people. Also, socially-mediated forms of literacy are especially important among subjects who are disinterested in ICTs. In the case of professional literacy, the type of techno-dispositions and digital skills acquired are relevant because they can be transferred to other fields of activity, working as a secondary techno-socialization process which is added to previous individualized forms of literacy. In the case of social support, subjects can deploy digital tasks beyond their internalized digital skills.

Summing up, the main contribution of the article to digital inclusion research is the presentation of a comprehensive model of digital literacy and its connection with the biographical process of socialization in the use of technological devices. Therefore, teaching specific sets of digital skills (top-down literacy) is insufficient to promote digital inclusion, since such competencies vary often and usually it is more important to be confident and familiar with the use of digital devices (bottom-up literacy). Hence, building up social spaces of use of digital devices in which people feel motivated to explore and experiment with ICTs, exchanging knowledge with others, is crucial to becoming familiar with digital tools. In Bourdieu's terms, familiarization and confidence are internalized in the habitus through techno-dispositions (Straubhaar, 2012) which can engender new courses of digitally-mediated action, enabling the possibility of internalizing new digital skills in the natural process of incorporation of digital devices in life.

\section{Conclusion}

In this article, we have shown how digital inclusion cannot be reduced by just granting material access to digital equipment and tools-the first-level digital divide (Compaine, 2001) - or by increasing people's level of digital skills-the second-level digital divide, referring to forms of use (Hargittai, 2002; van Deursen \& van Dijk, 2014). It is also necessary to generate a feasible environment of technological domestication in which people feel at ease in the use of digital technologies. Digital literacy cannot be achieved just by promoting intense spaces of learning structured sets of skills, since most of these competencies are acquired during long-term processes of domestication of digital devices (Silverstone, 1993). Consequently, digital inclusion policies should also aim to engender interests, motivations, and technodispositions (Rojas et al., 2012) among users, since the main barriers of digital performance are usually motivational (Reisdorf \& Groselj, 2017) and emotional (Huang et al., 2015): Past bad experiences of use affect current digital practices, and people who are unfamiliar with digital devices become more and more excluded from the potential opportunities of the information society. Regarding this, certain sociodemographic asymmetries have been identified. By gender, men are usually more motivated and confident regarding digital devices, although these differences are more blurred in the case of younger and better-educated respondents. By age group, among older respondents, digital asymmetries are much wider, although in the case of younger respondents the variety of forms of technological domestication experience since childhood is wider. In the case of medium-age respondents, socialization linked to personal computers stands out. Finally, higher education is associated with better computer proficiency, particularly due to the effect of professional literacy associated with the university and the potential access to more digitalized jobs.

Moreover, the lack of a long-term biography of unconscious literacy associated with digital devices is one of the main factors of digital exclusion, even when material accessibility to such equipment is granted. In these cases, professional literacy is one of the main ways of promoting digital literacy, since it allows people to incorporate digital dispositions and become familiar with technological devices, rather than just learning a structured formal set of digital skills-like what many formative courses are oriented towards. Also, the effect of social support is ambivalent: It empowers already motivated subjects who mobilize their social networks to increase their digital knowledge, but it disempowers people who perceive a lack of confidence and ability to learn new skills and who are only interested in delegating difficult tasks rather than in learning how to complete them. This recalls the stratification theory (Lupač, 2018; Ragnedda, 2017), which the third-level digital divide theory is built on. This theory suggests that social inequalities are magnified in the digital realm by the differential outcomes 
and benefits that people obtain from their distinctive appropriation of technology to increase their life chances. In conclusion, to promote digital inclusion, we need to focus less on top-down spaces of literacy-structured sets of skills-and more on generating bottom-up social spaces of digital practice in which people feel at ease (Sefton-Green et al., 2009). Thus, digital literacy policies should promote digitally mediated spaces of interaction in which people can become confident, familiar, and motivated towards the use of ICTs: This is the only way to incorporate strong techno-dispositions (Straubhaar, 2012) which could be used to learn new digital competencies under the diverse and mutable requirements of information society.

\section{Acknowledgments}

This article is funded by the Complutense University of Madrid under the project "Estudios sobre la Desigualdad Social/Social Inequality Studies" (reference FEI-EU-17-24).

\section{Conflict of Interests}

The author declares no conflict of interest.

\section{References}

Alonso, L. E. (1998). La mirada cualitativa en sociología [The qualitative look in sociology]. Madrid: Fundamentos.

Antonio, A., \& Tuffley, D. (2014). The gender digital divide in developing countries. Future Internet, 6(4), 673-687. https://doi.org/10.3390/fi6040673

Bolin, G. (2018). Generational analysis as a methodological approach to study mediatised social change. In S. Taipale, T.-A. Wilska, \& C. Gilleard (Eds.), Digital technologies and generational identity: ICT usage across the life course (pp. 23-36). Oxon: Routledge.

Bourdieu, P. (1979). La distinction. Critique sociale du jugement [Distinction: A social critique of the judgement of taste]. Paris: Éditions du minuit.

Castaño, C., Martín, J., \& Martínez, J. L. (2011). La brecha digital de género en España y Europa: Medición con indicadores compuestos [The digital divide in Spain and Europe: Measurement about composed indexes]. Revista Española de Investigaciones Sociológicas, 136, 127-140. https://doi.org/10.5477/cis/reis. 136.127

Compaine, B. (2001). The digital divide. Facing a crisis or creating a myth? Boston, MA: MIT Press.

Courtois, C., \& Verdegem, P. (2016). With a little help from my friends: An analysis of the role of social support in digital inequalities. New Media and Society, 18(8), 1508-1527. https://doi.org/10.1177/ 1461444814562162

Dutton, W. H., \& Reisdorf, B. C. (2019). Cultural divides and digital inequalities: Attitudes shaping Inter- net and social media divides. Information Communication and Society, 22(1), 18-38. https://doi.org/ 10.1080/1369118X.2017.1353640

Erstad, O. (2011). Citizens navigating in literate worlds: The case of digital literacy. In M. Thomas (Ed.), Deconstructing digital natives: Young people, technology, and the new literacies (pp. 99-118). New York, NY: Routledge.

Ghobadi, S., \& Ghobadi, Z. (2015). How access gaps interact and shape digital divide: A cognitive investigation. Behaviour \& Information Technology, 34(4), 330-340. https://doi.org/10.1080/0144929X.2013.833650

Haight, M., Quan-Haase, A., \& Corbett, B. A. (2014). Revisiting the digital divide in Canada: The impact of demographic factors on access to the Internet, level of online activity, and social networking site usage. Information, Communication \& Society, 17(4), 503-519. https://doi.org/10.1080/1369118X. 2014.891633

Hargittai, E. (2002). Second-level digital divide: Differences in people's online skills. First Monday, 7(4), 1-14.

Huang, K.-T., Robinson, L., \& Cotten, S. R. (2015). Mind the emotional gap: The impact of emotional costs on students learning outcomes. In L. Robinson, S. R. Cotten, J. Schulz, T. M. Hale, \& A. Williams (Eds.), Communication and information technologies annual. Digital distinctions and inequalities (Vol. 10, pp. 121-144). Bingley: Emerald Group.

Institute of Museum and Library Services, University of Washington, \& International City/County Management Association. (2012). Building digital communities: A framework for action. Washington, DC: Institute of Museum and Library Services. Retrieved from https://www.imls.gov/assets/1/AssetManager/ BuildingDigitalCommunities_Framework.pdf

Kvasny, L. (2006). The role of the habitus in shaping discourses about the digital divide. Journal of ComputerMediated Communication, 10(2). https://doi.org/ 10.1111/j.1083-6101.2005.tb00242.x

Lasén, A., \& Casado, E. (2014). Presentación: Convergencias y controversas en torno a las mediaciones tecnológicas de lo ordinario [Presentation: Convergences and controversies about technological mediation of the ordinary]. In A. Lasén \& E. Casado (Eds.), Mediaciones tecnológicas. Cuerpos, afectos y subjetividades (pp. 7-18). Madrid: Centro de Investigaciones Sociológicas.

Lee, H., Park, N., \& Hwang, Y. (2015). A new dimension of the digital divide: Exploring the relationship between broadband connection, smartphone use and communication competence. Telematics and Informatics, 31(1), 45-56. https://doi.org/10.1016/j.tele. 2014.02.001

Livingstone, S. (2008). Internet literacy: Young people's negotiation of new online opportunities. In T. McPherson (Ed.), Digital youth, Innovation and the Unexpected (pp. 101-122). Cambridge, MA: The MIT Press. 
Lupač, P. (2018). Beyond the digital divide. Contextualizing the information society. Bingley: Emerald Publishing Limited.

Mariën, I., \& Prodnik, J. A. (2014). Digital inclusion and user (dis)empowerment: A critical perspective. Info, 16(6), 35-47. https://doi.org/10.1108/info-07-20140030

Pearce, K. E., \& Rice, R. E. (2013). Digital divides from access to activities: Comparing mobile and personal computer internet users. Journal of Communication, 63(4), 721-744. https://doi.org/10.1111/jcom. 12045

Ragnedda, M. (2017). The third digital divide: $A$ Weberian approach to digital inequalities. New York, NY: Routledge.

Ragnedda, M., Ruiu, M. L., \& Addeo, F. (2019). Measuring digital capital: An empirical investigation. New Media and Society. Advance online publication. https:// doi.org/10.1177/1461444819869604

Reisdorf, B. C., \& Groselj, D. (2017). Internet (non-)use types and motivational access: Implications for digital inequalities research. New Media and Society, 19(8), 1157-1176. https://doi.org/10.1177/ 1461444815621539

Robinson, L. (2009). A taste for the necessary. Information, Communication \& Society, 12(4), 488-507. https://doi.org/10.1080/13691180902857678

Rojas, V., Straubhaar, J., Spence, J., Roychowdhury, D., Okur, O., Piñon, J., \& Fuentes-Bautista, M. (2012). Communities, cultural capital and digital inclusion. Ten years of tracking techno-dispositions and technocapital. In J. Straubhaar, J. Spence, Z. Tufekci, \& R. G. Lentz (Eds.), Inequality in the technopolis: Race, class, gender, and the digital divide in Austin (pp. 223-264). Austin, TX: University of Texas.

Sefton-Green, J., Nixon, H., \& Erstad, O. (2009). Review- ing approaches and perspectives on "digital literacy." Pedagogies: An International Journal, 4(2), 107-125. https://doi.org/10.1080/15544800902741556

Silverstone, R. (1993). Domesticating the revolution: Information and communication technologies and everyday life. Aslib Proceedings, 45(9), 227-233. https://doi.org/10.1108/eb051328

Straubhaar, J. (2012). Conclusion. In J. Straubhaar, J. Spence, Z. Tufekci, \& R. G. Lentz (Eds.), Inequality in the technopolis: Race, class, gender, and the digital divide in Austin (pp. 265-277). Austin, TX: University of Texas.

Valles, M. S. (2014). Entrevistas cualitativas [Qualitative interviews]. Madrid: Centro de Investigaciones Sociológicas.

van Deursen, A., Courtois, C., \& van Dijk, J. (2014). Internet skills, sources of support, and benefiting from Internet use. International Journal of HumanComputer Interaction, 30(4), 278-290. https://doi. org/10.1080/10447318.2013.858458

van Deursen, A., \& van Dijk, J. (2013). The digital divide shifts to differences in usage. New Media \& Society, 16(3), 507-526. https://doi.org/10.1177/ 1461444813487959

van Deursen, A., \& van Dijk, J. (2014). Digital skills. Unlocking the information society. New York, NY: Palgrave Macmillan.

van Deursen, A., \& van Dijk, J. (2015). Toward a multifaceted model of internet access for understanding digital divides: An empirical investigation. The Information Society, 31(5), 379-391. https://doi.org/ 10.1080/01972243.2015.1069770

Wengraf, T. (2001). Qualitative research interviewing: Biographic narrative and semi-structured methods. London: Sage.

\section{About the Author}

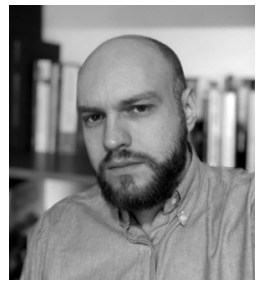

Daniel Calderón Gómez, PhD in Sociology and Anthropology (2019) at the Complutense University of Madrid, is member of the group of Social Inequality Studies (UCM, 2018-2020) and the RESCuE project team on "Citizens' Resilience in Times of Crisis" (EU 7th Framework Programme, 2015-2017). He also worked as a Pre-Doctoral researcher at the Department of Social Anthropology and Social Psychology (UCM, 2015-2019). His research interests include digital divide studies, information and communication technologies, sociology of youth, and social stratification. 